\title{
Collecting Systematic, Introspective Reports of Pharmacological Effects and Side-Effects
}

\author{
Gopal P. Sarma, $\mathrm{PhD}^{1}$ \\ ${ }^{1}$ School of Medicine, Emory University, Atlanta, GA, USA
}

\begin{abstract}
The study of subjective, first-person experience is a topic with both philosophical and practical implications. In this article, I discuss the value of collecting a critical mass of prose or verbal descriptions of introspectively determined, subjective effects of pharmacological agents. I suggest that datasets of introspective reports fit in the modern research landscape at the intersection of biomedical informatics and the emerging discipline of contemplative neuroscience. I compare the current proposal to Descriptive Experience Sampling (DES), discuss relevant methodological and conceptual issues in the study of introspection, and provide a list of questions for directing future investigation.
\end{abstract}

\section{Introduction}

Pharmacology is a cornerstone of modern medicine. To minimize the likelihood of drugs with adverse side effects reaching the public, multiple stages of safeguards, including animal models and post-marketing observational studies are used.

Drugs with side effects can broadly (and informally) be divided into two categories. Those which are toxic and cause severe physiological damage to an individual (or a fetus in utero, as in the case of thalidomide), and those whose side effects are non-lethal, but which are sufficiently unpleasant to make the drug an inviable candidate to be released to the market. For instance, a pain killer which completely attenuates migraines and causes severe but temporary abdominal pain is unlikely to be preferred over one that simply reduced the intensity of the migraine by $60 \%$ and had no other adverse effects.

Philosophically, there is a clear difference between these two categories. The first consists of effects that can be objectively verified by a third party observer, whereas the latter consists of introspective reports from the subject. In other words, this category consists of side effects (or simply effects) which relate to the inner experience of taking a drug. Consequently, to the extent that we have any information about the subjective experience of pharmacological effects and side-effects, they are restricted to cases of severe, or at the very least negative and unpleasant, side-effects. However, it is worth considering if there might be benefits to extending this knowledge-base to include more subtle effects of a drug (whether psychoactive or otherwise) and to make introspection a significant tool of investigation in modern pharmacology.

The present article relates to two contemporary areas of modern science and medicine. The first is the rapidly growing discipline of contemplative neuroscience. The aim of this discipline is to take an individual's subjective experience as the basic object of scientific scrutiny. Whether in the context of fundamental research pursued by philosophers and cognitive scientists, or with the aim of introducing introspective ideas into medical practice at centers for integrative medicine, many introspective traditions from around the world are finding their way into modern science [Barnes et al., 2004, Brown and Ryan, 2003, Carlson et al., 2003, Davidson et al., 2003, Kabat-Zinn, 2003, Lazar et al., 2005, Lutz and Thompson, 2003, Lutz et al., 2004, 
Ott et al., 2006, Shapiro et al., 1998, Harrington and Zajonc, 2008].

The second is the use of modern machine learning and data analytic tools in the biomedical research pipeline. The massive size of the biomedical literature, the existence of forums on the Internet where individuals report side-effects, and the availability of datasets of search queries, have enabled researchers to use completely data-driven techniques to uncover effects that were overlooked by the traditional methods of pharmacology and pharmaco-epidemiology [Bishop, 2007, Abu-Mostafa et al., 2012, Chen et al., 2007, Tatonetti et al., 2012, Percha et al., 2012] $]^{1}$.

The synthesis of these efforts would be large databases consisting of prose descriptions of introspective reports of pharmacological agents. These databases would not need to be restricted to drugs whose primary function is psychoactive. The thesis of the present article is that such databases would be valuable for all categories of drugs, whether pain killers, chemotherapy, statins, or herbal supplements. In addition to detailed descriptions, there are a range of simple questions one can easily ask about the internal experience of drugs that could prove to be useful when collected at a large scale. For instance, determining which compounds have introspectively noticeable effects at all is the most basic question one can ask. I consider this and other questions in the subsequent sections.

\section{Contemplative Neuroscience}

As pharmacology needs little introduction as a discipline, I begin with a brief discussion of contemplative neuroscience (or contemplative studies) and an overview of some contemporary research. The fundamental aim of these emerging topics of study is to bridge the knowledge of introspective traditions with modern neuroscience. Many areas of modern psychology and cognitive science study introspection and subjective experience to limited degrees. However, the aim of contemplative neuroscience is to take individuals' subjective experience and the dimensions of their inner life as the basic objects of scientific scrutiny [Varela, 1996, Harrington and Zajonc, 2008, Lutz and Thompson, 2003].

Introspection is not a new idea, and much of the knowledge that has driven modern contemplative neuroscience originates from traditions that extend back many centuries. These traditions include yoga, Tai Chi, Zen, and Tibetan Buddhism, to name just a few. While those from medically oriented communities are often interested in how contemplative techniques can influence patient outcomes and quality of life, particularly in the case of chronic pain or terminal illnesses [Ott et al., 2006, Sherman et al., 2005], a substantial amount of research has also taken place in the basic research setting. Modern imaging techniques and electrophysiology, such as fMRI, MEG, and EEG, have played a crucial role in these areas of research, allowing investigators to characterize the long and short-term changes taking place in the brains of advanced meditators who have devoted many thousands of hours to the practice of introspective techniques, or short-term interventions in novices. [Davidson et al., 2003, Lazar et al., 2005, Lutz et al., 2004].

However, in spite of the substantial growth of contemplative neuroscience, it is still a subject in its infancy, and there is certainly low-hanging fruit which has yet to be examined. In the remainder of the article, I will argue for the integration of pharmacology, introspection, and data-science as an important avenue for fundamental research.

\footnotetext{
${ }^{1}$ There are also commercial efforts taking advantage of these techniques, an area that is likely to grow substantially in the coming years. See, for example, Iodine, which uses crowdsourcing and predictive models to help patients find appropriate medications for their conditions with maximal efficacy and minimal side-effects.
} 


\section{Motivating Questions}

The primary aim of this article is to advocate for a certain type of study, or data collection endeavor. While the idea is simply stated, a number of conceptual and methodological questions naturally arise that would need to be addressed before proceeding further. Therefore, as a means to communicate this perspective, I have included below a list of questions to motivate additional investigation.

I have divided the questions into two categories. The first consists of basic questions about the subjective effects of pharmacological agents, i.e. what the late philosopher and contemplative neuroscience pioneer Francisco Varela termed "neuro-phenomenology" [Lutz and Thompson, 2003, Varela, 1996]. The second consists of methodological questions that might arise in attempting to collect this data.

\section{Fundamental Questions}

- What is the minimum dosage at which a drug is noticeable?

- What exogenous factors influence a person's ability to notice a drug? What about drug-drug interactions? To what extent is a person's ability to notice a drug related to their genotype for enzymes in the cytochrome P450 system, such as CYP2D6 [Atkinson Jr et al., 2012]?

- Is there a physiological analogue to perceptual adaptation [Moradi et al., 2005]? That is, are there certain drugs which make others more noticeable? For example, is someone who just took a large dose of caffeine more likely to notice a microdose of alcohol?

- In a large group of subjects, what is the distribution of minimum values at which a drug is noticeable? Can we explain why the distribution looks the way it does?

- Are there drugs, such as ordinary pain killers, that are not noticeable even at high dosages? That is, if a subject who was not in any pain took an ordinary pain killer (i.e., one that is not an opiate), would they be able to notice it? What is the minimum dosage?

- Suppose we have two drugs which are both noticeable at some level. Are they discriminable from each other? That is, if a person is told that they are being given either drug A or drug B at the minimum noticeable dose, are they able to detect which one they were administered? How reliably? What accounts for the variation in a person's ability to detect a drug when administered blindly?

- What is the best predictor of the level at which a drug is noticeable to an individual?

- How do body-oriented introspective practices, such as Tai Chi, yoga, Alexander Technique, among others, impact a person's ability to notice small doses of a drug [Gard et al., 2011, Kerr et al., 2008, Mehling et al., 2005, Sherman et al., 2005, Price, 2005, Ives, 2003, Klein et al., 2014, Mehling, 2001, Smith et al., 1999]? How do these relate to meditation [Lazar et al., 2005, Harrington and Zajonc, 2008]?

- Suppose that we are able to determine, for a given individual, the minimum noticeable dosages for a wide variety of drugs. Do these values form a distinct signature of the person? Are there are other physiological variables that such a signature is a strong predictor of?

- Suppose that we have detailed prose descriptions of the effects of many classes of drugs from many individuals. Do they form natural clusters? How do the clusters determined informally by investigators relate to those identified by standard clustering techniques from machine learning [Bishop, 2007, AbuMostafa et al., 2012]? Do the clusters in any way relate to the chemical structure or properties of the compounds? 


\section{Methodological Questions}

- What are the ethical issues that arise in collecting introspective reports of pharmacological agents? How will collecting this data fit into existing practices overseen by Institutional Review Boards?

- How reliable are introspective reports? What is the variation over time of a given individual's reports? Do past experiences shape a person's response to microdoses of a drug?

- Are there methods for eliciting more reliable introspective reports? What about techniques for training a person to notice the effects of a drug?

- Are there other modalities for recording introspective observations? For instance, as machine learning and data analytic capabilities become increasingly powerful and capable of handling larger and larger datasets, will audio and video recordings prove to be a more powerful modality? Or is the additional information mostly extraneous relative to prose descriptions?

- Do we have an adequate vocabulary for capturing the subtle effects of a drug on the body and mind? Are the insights of long-standing introspective traditions relevant to improving a person's ability to capture the internal experience of a particular drug?

- Collecting the necessary data, such as determining the minimum noticeable dosages of a wide variety of compounds, would be a fairly involved process, and likely have more in common with extended sleep studies in a hospital setting, rather than the short, and easily administered psychophysics experiments conducted at university cognitive science departments. What are the practical obstacles to conducting studies of this kind? What lessons can we learn from related studies that have been conducted before?

\subsection{Descriptive Experience Sampling}

Some of the above questions overlap with existing methodologies and research efforts in contemplative neuroscience. I will briefly discuss one of them, namely, the technique of Descriptive Experience Sampling (DES). The aim of DES is to capture the internal experiences of individuals in a minimally invasive, naturalistic setting and to provide a large body of information for advancing contemplative neuroscience. In DES, subjects wear a device which beeps at random intervals. When the device beeps, subjects are instructed to write down everything they were experiencing in that moment [Hurlbut and Schwitzgebel, 2007, Engelbert and Carruthers, 2011].

The present proposal is related to DES, although the aim is to capture a much narrower type of information, rather than the entirety of what a person is experiencing in a given moment. It is worth mentioning several of the criticisms that have been raised about DES and how they relate to collecting introspective information about pharmacological effects. Broadly speaking, DES has been criticized for producing reports of questionable validity after probing by an investigator. That is, people may in one moment report thinking one set of thoughts, or having one set of experiences, and then change their mind after the fact when asked pointed questions. In addition, some researchers have highlighted the difficulty subjects face in simultaneously experiencing and reporting a subjective state, and the inherent ambiguity in forming an explicit linguistic representation of a subjective experience [Hurlbut and Schwitzgebel, 2007, Engelbert and Carruthers, 2011].

The questions I have raised above may circumvent the possible complications of DES. In particular, critics of DES grant that the technique can be reliable when restricted to gross aspects of sensory experience, such as simply noticing that one is in pain, or observing the color of an object. A number of the above questions involve merely detecting that a microdose of a compound had been administered relative to a placebo, and not characterizing the evolution of ongoing mental states. If, on the other hand, one attempted to understand

how a particular drug impacted a person's inner life in a naturalistic setting - certainly an important lines of questioning - then the complications of the DES methodology would be relevant. 


\section{Conclusion}

The aim of this article is to argue that research integrating data science, contemplative neuroscience, and pharmacology is of both philosophical and practical significance. To motivate possible research directions that lie at the intersection of these three disciplines, I propose several questions relating to the subjective experience of pharmacological agents. For instance, understanding the reliability of introspective observation and recall has been a significant area of debate in contemplative neuroscience, and continues to be an active area of research and investigation. In situations where ancient contemplative practices have insights that are relevant to modern science, barriers of cross-cultural understanding must also be traversed. The questions that I have asked above relating to minimum noticeable dosages (MNDs) circumvent some complications that arise in collecting reliable introspective reports.

Large data sets of introspective reports of pharmacological agents - whether prose descriptions or binary variables (e.g. noticeable / not-noticeable) - will be useful for a variety of reasons. Practically, such data sets will be a valuable resource for exploratory data analysis. For instance, knowledge of the minimum noticeable dosages for a wide variety of compounds may allow us to predict drug-drug interactions. Predictive models developed using data from introspective reports could enable us to predict non-lethal or unpleasant side-effects of novel drug. A large number of minimum noticeable dosages might encode a distinct, physiological signature of a person. If so, such a signature could be a valuable predictor of other medically important parameters.

Finally, exploratory data analysis on large bodies of introspective reports may play an important role in advancing the fundamental methodology and basic knowledge of contemplative neuroscience. The systematic study of introspection is in its nascent stages and many basic methodological issues are just now being explored. There is much to be gained from additional interaction between these communities and medical investigators. Furthermore, utilizing data science to analyze introspective reports by both lay individuals as well as those highly trained in introspective techniques may allow us to move beyond uncovering unpleasant side-effects, develop a more sophisticated understanding of inner experience, and systematize taxonomies from prose descriptions.

\section{Acknowledgments}

I would like to thank Adam Safron, Erik Reinersten, and Seshu Sarma for feedback on the manuscript.

\section{References}

Y. Abu-Mostafa, M. Magdon-Ismail, and L. H.L. Learning from Data. AMLBook, Pasadena, 2012.

A. J. Atkinson Jr, S.-M. Huang, J. J. Lertora, and S. P. Markey. Principles of Clinical Pharmacology. Academic Press, 2012.

V. A. Barnes, H. C. Davis, J. B. Murzynowski, and F. A. Treiber. Impact of meditation on resting and ambulatory blood pressure and heart rate in youth. Psychosomatic Medicine, 66(6):909-914, 2004.

C. Bishop. Pattern Recognition and Machine Learning. Springer, Berlin, 2007.

K. W. Brown and R. Ryan. The Benefits of Being Present: Mindfulness and Its Role in Psychological Well-Being. Journal of Personality and Social Psychology, 84(4):822-48, 2003.

S. Carlson, L. E., K. D. M., Patel, , and E. Goodey. Mindfulness-based stress reduction in relation to quality of life, mood, symptoms of stress, and immune parameters in breast and prostate cancer outpatients. Psychosomatic Medicine, 65(4):571-581, 2003. 
Y. Chen, L. Pedersen, C. W.W., and O. J. Drug Exposure Side Effects from Mining Pregnancy Data. ACM SIGKDD Explorations Newsletter, 9(1):22-29, 2007.

R. Davidson, J. Kabat-Zinn, J. Schumacher, M. Rosenkrantz, D. Muller, and S. F. Santorelli. Alterations in brain and immune function produced by mindfulness meditation. Psychosomatic Medicine, 65(4):564-570, 2003.

M. Engelbert and P. Carruthers. Descriptive Experience Sampling: What is it good for? Journal of Consciousness Studies, 18(1):pp.130-149, 2011.

T. Gard, N. Brach, B. K. Hölzel, J. J. Noggle, L. A. Conboy, and S. W. Lazar. Effects of a yoga-based intervention for young adults on quality of life and perceived stress: The potential mediating roles of mindfulness and self-compassion. The Journal of Positive Psychology, 7(3):pp.165-175, 2011.

A. Harrington and A. Zajonc. The Dali Lama at MIT. Harvard University Press, Cambridge, 2008.

R. Hurlbut and E. Schwitzgebel. Describing Inner Experience: Proponent Meets Skeptic. MIT Press, Cambridge, 2007.

J. C. Ives. Comments on the Feldenkrais Method: a dynamic approach to changing motor behavior. Res $Q$ Exerc Sport, 74(2):116-123, Jun 2003.

J. Kabat-Zinn. Mindfulness-Based Interventions in Context: Past, Present, and Future. Clinical Psychology: Science and Practice, 10(2):144-156, 2003.

C. E. Kerr, J. R. Shaw, R. H. Wasserman, V. W. Chen, A. Kanojia, T. Bayer, and J. M. Kelley. Tactile acuity in experienced Tai Chi practitioners: evidence for use dependent plasticity as an effect of sensoryattentional training. Experimental Brain Research, 188(2):pp. 317-22, 2008.

S. D. Klein, C. Bayard, and U. Wolf. The Alexander Technique and musicians: a systematic review of controlled trials. BMC Complement Altern Med, 14:414, 2014.

S. W. Lazar, C. E. Kerr, R. H. Wasserman, J. R. Gray, G. D. N., M. T. Treadway, McGarvey, Q. Metta, B. T., J. A. Dusek, H. Benson, S. L. Rauch, C. I. Moore, and B. Fischld. Meditation Experience is Associated with Increased Cortical Thickness. NeuroReport, 16(17):1893-1897, 2005.

A. Lutz and E. Thompson. Neurophenomenology: Integrating Subjective Experience and Brain Dynamics in the Neuroscience of Consciousness. Journal of Consciousness Studies, 10(9-10):21-52, 2003.

A. Lutz, L. Greischar, N. Rawlings, M. Riccard, and R. Davidson. Long-term Meditators self-induce high amplitude gamma synchrony during mental practice. Proceedings of the National Academy of Sciences, 101(46):16360-16373, 2004.

W. E. Mehling. The experience of breath as a therapeutic intervention - psychosomatic forms of breath therapy. A descriptive study about the actual situation of breath therapy in Germany, its relation to medicine, and its application in patients with back pain. Forsch Komplementarmed Klass Naturheilkd, 8 (6):359-367, Dec 2001.

W. E. Mehling, Z. DiBlasi, and F. Hecht. Bias control in trials of bodywork: a review of methodological issues. J Altern Complement Med, 11(2):333-342, April 2005.

F. Moradi, C. Koch, and S. Shimojo. Face adaptation depends on the seeing the face. Neuron, 45(1):169-175, 2005.

M. Ott, R. Norris, and S. Bauer-Wu. Mindfulness Meditation for Oncology Patients: A Discussion and Critical Review. Integrative Cancer Therapies, 5(2):98-108, 2006. 
B. Percha, Y. Garten, and R. B. Altman. Discovery and Explanation of Drug-Drug Interactions via Text Mining. Proceedings of the Pacific Symposium on Biocomputing, pages pp. 410-421, 2012.

C. Price. Body-oriented therapy in recovery from child sexual abuse: an efficacy study. Altern Ther Health Med, 11(5):46-57, 2005.

S. Shapiro, G. Schwartz, and G. Bonner. Effects of Mindfulness-Based Stress Reduction on Medical and Premedical Students. Journal of Behavioral Medicine, 21(6):581-599, 1998.

K. J. Sherman, D. C. Cherkin, J. Erro, D. L. Miglioretti, and R. A. Deyo. Comparing yoga, exercise, and a self-care book for chronic low back pain: a randomized, controlled trial. Ann. Intern. Med., 143(12): 849-856, Dec 2005.

M. C. Smith, M. A. Stallings, S. Mariner, and M. Burrall. Benefits of massage therapy for hospitalized patients: a descriptive and qualitative evaluation. Altern Ther Health Med, 5(4):64-71, Jul 1999.

N. P. Tatonetti, Y. P. P., R. Daneshjou, and R. B. Altman. Data-Driven Prediction of Drug Effects and Interactions. Science Translational Medicine, 4(125):pp.125-131, 2012.

F. J. Varela. Neurophenomenology: A methodological remedy for the hard problem. Journal of Consciousness Studies, 3(4):330-349, 1996. 